\title{
Properties of multinomial lattices with cumulants for option pricing and hedging
}

\author{
Yuji Yamada \\ Graduate School of Business Sciences, University of Tsukuba, Tokyo, Japan 112-0012 \\ Email: yuji@gssm.otsuka.tsukuba.ac.jp \\ James A. Primbs \\ Management Science and Engineering, Stanford University, Stanford, CA 94305-4026 \\ Email: japrimbs@stanford.edu
}

\begin{abstract}
In this paper, we analyze properties of multinomial lattices that model general stochastic dynamics of the underlying stock by taking into account any given cumulants (or moments). First, we provide a parameterization of multinomial lattices, and demonstrate that mean, variance, skewness, and kurtosis of the underlying may be matched using five branches. Then, we investigate the convergence of the multinomial lattice when the basic time period approaches zero, and prove that the limiting process of the multinomial lattice that matches annualized mean, variance, skewness and kurtosis is given by a compound Poisson process. Finally, we illustrate the effect of higher order moments in the underlying asset process on the price of derivative securities through numerical experiments using the multinomial lattice, and provide a comparison with jump-diffusion models.
\end{abstract}

Keywords: Multinomial lattice, Cumulants, Excess kurtosis and skewness, Compound Poisson process, Volatility smile

\section{Introduction}

It is widely recognized today that there is a non-negligible discrepancy between the Black-Scholes model and real market behavior, which appears as the "smile effect" or "implied volatility smile" in option markets. With empirical evidence that implied volatility increases for in-the-money or outof-the-money options, both theoretical and empirical research on models that exhibit a smile effect and/or heavy tail phenomena has been especially active since the late 80 's $[9,10,12,11,20,29]$, and option valuation techniques have been extended to more realistic assumptions in a number of ways for the underlying stock processes (e.g., [1, 2, 4, 7, 16, 17, 21, 24, 25, 22]), distributions (e.g., $[6,20,26,27,31,30]$ ), and markets (e.g., [15, 32, 33]).

In this body of literature, there is a group of research related to discrete models and their generalization. This type of research may be categorized into two approaches: The first approach is to formulate a general continuous time model and discretize it to solve pricing problems $[1,5,7,21]$. The second approach is to generalize the standard discrete model (such as the standard binomial tree or

trinomial tree models $[8,18]$ ), directly, to incorporate real market behavior characterized as smiles or heavy tails $[10,29,30]$. Although continuous models may sometimes provide a closed form solution 
which can be solved analytically, the advantage in considering discrete models is that we can solve a wider class of problems (such as American options or some Exotics) for option pricing and hedging efficiently by using numerical techniques.

The objective of this paper is to provide another discrete model based on multinomial lattices with cumulants, and analyze its properties. To achieve this objective, we first model general stochastic dynamics of the underlying stock using multinomial lattices which take into account any given cumulants (or moments). We demonstrate that the mean, variance, skewness, and kurtosis of the underlying may be matched using five branches, and prove the positivity condition to be satisfied for probabilities to construct the multinomial lattice. The convergence of the multinomial lattice is then investigated when the basic time period approaches zero, and it is shown that the limiting distribution of the multinomial lattice that matches annualized mean, variance, skewness and kurtosis is given by that of a compound Poisson process. We will illustrate the effect of higher order cumulants in the underlying asset process on the price of derivative securities through numerical experiments using the multinomial lattice, and make a comparison with jump-diffusion models. The relation between the multinomial lattice parameterization and the finite difference method for partial differential equations is also discussed.

Note that the ideas of a multinomial lattice and moment matching originate from the pioneering work of [7] using the binomial lattice model, and a number of extensions have been proposed since then for modeling stock (or option) dynamics [1, 5, 8, 21, 29, 30] or interest rate dynamics [19, 23]. Therefore, providing a multinomial lattice parameterization that matches moments itself might not be innovative. However it should be mentioned that the multinomial lattice parameterization of this paper has some advantage over previous work in the sense that the parameterization proposed here is simple and tractable, and is easy to implement. Moreover, the theoretical analysis in the paper allows us to guarantee convergence and the positivity condition, which might be useful for practitioners as well.

This paper is organized as follows: In Section 2, we provide a parameterization of multinomial lattices and show the positivity condition for the probabilities. Then we apply mean square hedging to evaluate the price of options in Section 3, and analyze the limiting property of the multinomial lattice in Section 4. The comparison between the multinomial lattice and the jump-diffusion model is made in Section 5. Section 6 offers some concluding remarks.

\section{Multinomial lattice model}

\subsection{Stock dynamics}

In this paper, we will model the underlying stock dynamics in the time interval $t \in\left[0, t_{N}\right]$, where traders are allowed to purchase and sell the stock at the equally spaced discrete times $t_{n}, n=$ $0,1, \ldots, N$ with $t_{0}=0$. We will denote the price of the stock at discrete times $t=t_{n}$ by $S_{n}, n=$ $0,1, \ldots, N$.

Assume that the stock price evolves randomly on an $L$ state lattice model (i.e., given the price of the stock at time $t_{n}, n=0, \ldots, N-1$, there are $L$ possible future prices that it can take at time $\left.t_{n+1}\right)$. Suppose that $u$ and $d$ satisfy $u>d>0$, then a multinomial lattice can be constructed by taking the $L$ possible future states for $S_{n+1}$ from $S_{n}$ as

$$
S_{n+1}=u^{L-l} d^{l-1} S_{n}, l=1, \ldots L
$$


with probabilities $p_{l}, l=1, \ldots L$, satisfying $p_{1}+\cdots+p_{L}=1$. In this case, the stock may achieve $n(L-1)+1$ possible prices at time $t=t_{n}, n=0, \ldots, N$ given by

$$
S_{n}^{(k)}=u^{n(L-1)+1-k} d^{k-1} S_{0}, k=1, \ldots, n(L-1)+1 .
$$

Note that $u$ and $d$ may be thought of up and down factors at each step. Also, it can be shown that the multinomial lattice still recombines even if $u$ and $d$ are time-dependent when $u_{n} / d_{n}=c$ is satisfied for some constant $c>1$, where $u_{n}$ and $d_{n}, n=0,1, \ldots, N-1$, are up and down factors at each time step. An example of a multinomial lattice is depicted in Fig. 2.1 with a recombining condition $u_{n} / d_{n}=c$.

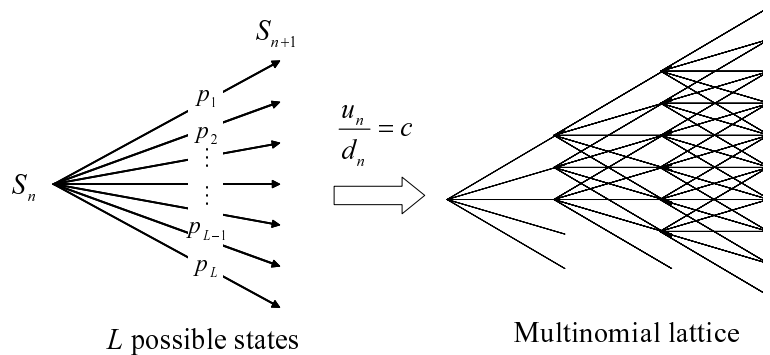

Fig. 2.1: Multinomial lattice model

\subsection{Parameterization of multinomial lattices}

In the multinomial lattice model, we need to determine the up and down rates $u$ and $d$, and the probabilities $p_{1}, \ldots, p_{L}$ to fit actual market data as closely as possible. This may be done by taking the cumulant (or moment) information of the underlying stock into account, and we have provided a parameterization of multinomial lattice random walks to match given cumulant information [34, 35]. Here we will briefly summarize those results, and then provide further discussion.

Let us introduce the log stock price at time $t_{n}$ as

$$
X_{n}:=\ln S_{n}, \quad n=0,1, \ldots, N .
$$

Then (2.1) may be rewritten as

$$
X_{n+1}-X_{n}=(L-l) \ln u+(l-1) \ln d, \quad l=1, \ldots L .
$$

Assume that the the $\log$ stock return is i.i.d., i.e., the moments of $X_{n+1}-X_{n}$ are the same for all $n=0, \ldots, N-1$, and that its $k$-th order cumulant is given as $c_{k}, k \geq 1$. Let the up and down rates, $u$ and $d$, be given as

$$
u:=\exp \left(\frac{\nu}{L-1}+\alpha\right), \quad d:=\exp \left(\frac{\nu}{L-1}-\alpha\right),
$$

where $L$ is the number of branches, and $\nu$ and $\alpha>0$ are real numbers. In this case, the first order 
cumulant (i.e., mean) of $X_{n+1}-X_{n}$ may be computed as

$$
\begin{aligned}
& \sum_{l=1}^{L} p_{l}\{(L-l) \ln u+(l-1) \ln d\} \\
= & \sum_{l=1}^{L} p_{l}\left\{(L-l)\left(\frac{\nu}{L-1}+\alpha\right)+(l-1)\left(\frac{\nu}{L-1}-\alpha\right)\right\} \\
= & \nu
\end{aligned}
$$

The above condition ensures that the first order cumulant of $X_{n}-X_{n-1}$ is matched using $\nu$, i.e., $\nu$ denotes the mean of $X_{n}-X_{n-1}$.

Similar to (2.6), one can compute the $k$-th order central moment ${ }^{1}$ as

$$
\begin{aligned}
& \sum_{l=1}^{L} p_{l}\{(L-l) \ln u+(l-1) \ln d-\nu\}^{k} \\
= & \sum_{l=1}^{L} p_{l}\left\{(L-l)\left(\frac{\nu}{L-1}+\alpha\right)+(l-1)\left(\frac{\nu}{L-1}-\alpha\right)-\nu\right\}^{k} \\
= & \sum_{l=1}^{L} p_{l}(L-2 l+1)^{k} \alpha^{k} .
\end{aligned}
$$

Let $\mu_{k}(k \geq 2)$ be the $k$-th order central moment of $X_{n}-X_{n-1}$, where the $k$-th order central moment may be computed by using the following relation:

$$
\mu_{k}=\sum_{j=1}^{k}\left(\begin{array}{c}
k-1 \\
j-1
\end{array}\right) c_{j} \mu_{k-j} .
$$

Then a moment matching condition would require that

$$
\sum_{l=1}^{L} p_{l}(L-2 l+1)^{k} \alpha^{k}=\mu_{k} .
$$

More generally, we have (2.9) for all $k \geq 0$ by setting $\mu_{0}=1$ and $\mu_{1}=0$ because the sum of $p_{l}, l=1, \ldots, L$, is 1 and the first order central moment may be considered as 0 .

Now, we would like to compute $p_{1}, \ldots, p_{L}$ and $\alpha$ that satisfy (2.9). Assume that the first $m$ cumulants are given. In this case, the first $m$ central moments are computed using (2.8), and there are $m+1$ constraints for $L$ plus 1 unknown parameters, $p_{1}, \ldots, p_{L}$ and $\alpha$. If $\alpha>0$ is fixed a priori, $p_{1}, \ldots, p_{L}$ can be computed by solving $m+1$ linear equations. In this case, we need at least $L=m+1$ branches to guarantee the existence of a feasible solution. After computing $p_{1}, \ldots, p_{L}, \alpha>0$ may be adjusted such that all the probabilities are positive. In particular, the first four cumulants are often used to characterize a given random variable or distribution because they provide important information such as the mean $\left(=c_{1}\right)$, variance $\left(=c_{2}\right)$, skewness, and excess kurtosis of the random variable, where skewness and excess kurtosis are given as

$$
\frac{c_{3}}{c_{2}^{3 / 2}}, \frac{c_{4}}{c_{2}^{2}},
$$

\footnotetext{
${ }^{1}$ Given the first $k$ cumulants, we can always compute the $k$-th order central moment. On the other hand, the $k$-th order cumulant may be computed using the first $k$ central moments.
} 
respectively. Here we provide a parameterization of the multinomial lattice to match the first four moments using five branches.

Let mean, variance, skewness, and excess kurtosis of $X_{n+1}-X_{n}$ be given as

$$
\nu, \sigma^{2}, s, \kappa
$$

Note that skewness $s$ and excess kurtosis $\kappa$ are zero for Gaussian random variables and that excess kurtosis is usually positive for most stock markets. In this paper, we will assume that $\kappa \geq 0$.

The probabilities $p_{l}, l=1, \ldots, 5$, can be calculated through the solution of five linear equations, and are given by

$$
\begin{aligned}
& p_{1}=\frac{\sigma^{2}}{384 \alpha^{4}}\left(-4 \alpha^{2}+4 s \sigma \alpha+(3+\kappa) \sigma^{2}\right) \\
& p_{2}=\frac{\sigma^{2}}{96 \alpha^{4}}\left(16 \alpha^{2}-2 s \sigma \alpha-(3+\kappa) \sigma^{2}\right) \\
& p_{3}=\frac{1}{64 \alpha^{4}}\left(64 \alpha^{4}-20 \sigma^{2} \alpha^{2}+(3+\kappa) \sigma^{4}\right) \\
& p_{4}=\frac{\sigma^{2}}{96 \alpha^{4}}\left(16 \alpha^{2}+2 s \sigma \alpha-(3+\kappa) \sigma^{2}\right) \\
& p_{5}=\frac{\sigma^{2}}{384 \alpha^{4}}\left(-4 \alpha^{2}-4 s \sigma \alpha+(3+\kappa) \sigma^{2}\right)
\end{aligned}
$$

In this formulation, we can confirm the following properties:

- The probabilities are unsymmetric if skewness is not zero $(s \neq 0)$, i.e.,

- positive (negative) skewness causes $p_{1}$ and $p_{4}$ to increase (decrease), and

- the corresponding probabilities $p_{5}$ and $p_{2}$ to decrease (increase) by an equal amount.

- The probability distribution of $X_{n}-X_{n-1}$ becomes leptokurtic (or heavy tailed) under positive excess kurtosis, i.e.,

$-p_{1}, p_{3}$ and $p_{5}$ increase with larger kurtosis, and

$-p_{2}$ and $p_{4}$ decrease if kurtosis increases.

Moreover, one can show that there exists $\alpha$ such that all the probabilities, $p_{l}, l=1, \ldots, 5$, are positive if and only if

$$
\kappa>3 s^{2}-3 .
$$

In the next subsection, we will discuss more detail about the above positivity condition. The convergence of the multinomial lattice when $N \rightarrow \infty$, or $\tau \rightarrow 0$, will be examined in Section 4, where $\tau$ is a basic time period defined as

$$
\tau:=\frac{t_{N}}{N}
$$

Remark 1 Although the multinomial lattice parameterization provided in this paper is as tractable as the standard binomial and trinomial lattices and may be extended to the multidimensional case, the computational effort might increase exponentially with respect to the dimension, similar to the other lattice models; This might be a limitation for these lattice models. However the multinomial lattices can be considered useful for relatively low-dimensional problems and be used to solve American option problems. 


\subsection{The positivity condition}

Consider the probabilities $p_{1}, \ldots, p_{5}$ in (2.11). Assume that $p_{1}, \ldots, p_{5}$ are strictly positive. By solving the simultaneous inequalities $p_{1}>0, \ldots, p_{5}>0$ with respect to $\alpha$, we obtain the following:

$$
\frac{\sigma}{16}\left(|s|+\sqrt{s^{2}+16(\kappa+3)}\right)<\alpha<\frac{\sigma}{2}\left(-|s|+\sqrt{s^{2}+\kappa+3}\right) .
$$

Since

$$
\frac{\sigma}{4} \sqrt{3+\kappa} \leq \frac{\sigma}{16}\left(|s|+\sqrt{s^{2}+16(\kappa+3)}\right)
$$

and

$$
\frac{\sigma}{2}\left(-|s|+\sqrt{s^{2}+(\kappa+3)}\right) \leq \frac{\sigma}{2} \sqrt{3+\kappa}
$$

hold for all $s \in \Re$, we see that a necessary condition of (2.14) is given as

$$
\frac{\sigma}{4} \sqrt{3+\kappa}<\alpha<\frac{\sigma}{2} \sqrt{3+\kappa}
$$

Let us replace $\alpha$ by

$$
\alpha=\sigma \sqrt{\frac{3+\kappa}{\beta}}, 4<\beta<16 .
$$

Then $p_{1}, \ldots, p_{5}$ in (2.11) may be rewritten as

$$
\begin{aligned}
& p_{1}=\frac{\beta}{384(3+\kappa)^{2}}((\beta-4)(3+\kappa)+4 s \sqrt{(3+\kappa) \beta}) \\
& p_{2}=\frac{\beta}{96(3+\kappa)^{2}}((16-\beta)(3+\kappa)-2 s \sqrt{(3+\kappa) \beta}) \\
& p_{3}=\frac{1}{64(3+\kappa)}\left(64(3+\kappa)-20 \beta+\beta^{2}\right) \\
& p_{4}=\frac{\beta}{96(3+\kappa)^{2}}((16-\beta)(3+\kappa)+2 s \sqrt{(3+\kappa) \beta}) \\
& p_{5}=\frac{\beta}{384(3+\kappa)^{2}}((\beta-4)(3+\kappa)-4 s \sqrt{(3+\kappa) \beta})
\end{aligned}
$$

We first note that $p_{3}>0,{ }^{\forall} \beta \in(4,16)$. From (2.17), we see that $p_{1}>0$ and $p_{5}>0$ hold if and only if

$$
\kappa>\frac{16 s^{2} \beta}{(\beta-4)^{2}}-3
$$

Similarly, $p_{2}>0$ and $p_{4}>0$ hold if and only if

$$
\kappa>\frac{4 s^{2} \beta}{(16-\beta)^{2}}-3
$$

Therefore $p_{1}>0, p_{2}>0, p_{3}>0$ and $p_{4}>0$ hold if and only if

$$
\kappa>\max \left[\frac{16 s^{2} \beta}{(\beta-4)^{2}}, \frac{4 s^{2} \beta}{(16-\beta)^{2}}\right]-3 .
$$

for any given $\beta \in(4,16)$.

Now, we will further examine the right hand side of the inequality in $(2.20)$. Because $\frac{16 s^{2} \beta}{(\beta-4)^{2}}$ monotonically decreases with $\beta \in(4,16)$ and $\frac{4 s^{2} \beta}{(16-\beta)^{2}}$ monotonically increases with $\beta \in(4,16)$, the 
right hand side of (2.20) takes its minimum if there exists an intersection between the two in the interval of $\beta \in(4,16)$. In fact,

$$
\frac{16 s^{2} \beta}{(\beta-4)^{2}}=\frac{4 s^{2} \beta}{(16-\beta)^{2}} .
$$

has the solution of $\beta=12$ which satisfies $\beta \in(4,16)$. Then we have

$$
\kappa>\max \left[\frac{16 s^{2} \beta}{(\beta-4)^{2}}, \frac{4 s^{2} \beta}{(16-\beta)^{2}}\right]-3 \geq 3 s^{2}-3 .
$$

where the equality holds when $\beta=12$, or

$$
\alpha=\sigma \sqrt{\frac{3+\kappa}{12}} .
$$

Finally, we obtain the following proposition:

Proposition 1 There exists $\alpha$ such that all the probabilities $p_{l}, l=1, \ldots, 5$, are positive if and only if

$$
\kappa>3 s^{2}-3 .
$$

and such a choice of $\alpha$ may be given as in (2.23).

Assume that $\alpha$ is given by (2.23). In this case, condition (2.11) simplifies to

$$
\begin{aligned}
& {\left[p_{1}, p_{2}, p_{3}, p_{4}, p_{5}\right]=} \\
& \quad\left[\frac{3+\kappa+s \sqrt{9+3 \kappa}}{4(3+\kappa)^{2}}, \frac{3+\kappa-s \sqrt{9+3 \kappa}}{2(3+\kappa)^{2}}, \frac{3+2 \kappa}{2(3+\kappa)}, \frac{3+\kappa+s \sqrt{9+3 \kappa}}{2(3+\kappa)^{2}}, \frac{3+\kappa-s \sqrt{9+3 \kappa}}{4(3+\kappa)^{2}}\right] .
\end{aligned}
$$

We can readily confirm that the probabilities are positive if (2.24) is satisfied.

\section{Mean square optimal hedging using multinomial lattices}

Before providing further discussion about the multinomial lattice, we will demonstrate a pricing algorithm using mean square optimal hedging (MSOH) on the multinomial lattice, which allows us to find an initial value of derivative securities including European and American options in a discrete market. Note that, in contrast to a binomial lattice case, the standard risk neutral pricing method may not be applied directly, since perfect replication is not possible on a multinomial lattice due to the incompleteness of the discrete market (except the binomial model) and as a result a unique risk neutral probability may not be obtained. MSOH provides an optimal solution in the mean square sense for such discrete models, and can be solved efficiently using dynamic programming[11, 13, 14, 15, 32, 33].

\subsection{Mean square optimal price}

Let $S_{n}$ be the price of the underlying stock at $t=t_{n}$ whose dynamics is expressed using a multinomial lattice. We will consider a self-financing portfolio which consists of the stock and a risk free bank account with fixed interest rate $r>0$. The portfolio value $\Omega_{n}(n=0 \ldots N)$ satisfies the following difference equation:

$$
\begin{aligned}
\Omega_{n+1} & =\Delta_{n} S_{n+1}+R\left(\Omega_{n}-\Delta_{n} S_{n}\right) \\
& =R \Omega_{n}+\Delta_{n}\left(S_{n+1}-R S_{n}\right), \quad n=0 \ldots N,
\end{aligned}
$$


where $\Delta_{n}$ is the number of shares of the stock held from $n$ to $n+1$, and $R:=1+r$.

The objective of mean square optimal hedging $(\mathrm{MSOH})$ is to optimally replicate or hedge the payoff of a European derivative security $V_{N}$ of maturity $N$ through a self-financing trading strategy with an adequate initial portfolio value $\Omega_{0}$. This involves solving the following optimization problem:

$$
\text { MSOH: } \min _{\Omega_{0}, \Delta_{0}, \ldots, \Delta_{N-1}} \mathbb{E}\left[\left(V_{N}-\Omega_{N}\right)^{2}\right]
$$

subject to the dynamics of the underlying stock and the portfolio.

This problem has been studied extensively [11, 13, 14, 15, 32, 33] and can be solved using dynamic programming. Although we only formulate the $\mathrm{MSOH}$ problem for a European call option, note that the same approach can be extended to other types of options, including exotics (such as barriers and compounds), and options with time optionality (such as Americans and Bermudans). The dynamic programming algorithm for MSOH only requires a change in the "boundary condition" corresponding to the appropriate option type, and proper discounting to account for the time value of different wealth balance cash flows.

Once the MSOH problem has been solved, the optimal initial portfolio value may be associated with the value of the option and provides the mean square optimal price, i.e.,

$$
V_{0}=\Omega_{0}
$$

Under this price, $\mathbb{E}\left(V_{N}-\Omega_{N}\right)=0$ is satisfied [13], and the objective function in MSOH gives the minimum variance of the hedging error. Therefore, in this situation, the MSOH problem can be thought of as minimizing the risk in the hedge as measured by the variance subject to a zero mean constraint. Note that, although it has been shown that the above "price" may sometimes lead to arbitrage opportunities (see the example by Schweizer [33]), we shall still refer to it as a price with the possibility of abuse, in keeping with mean-variance theory.

\subsection{Illustrative example}

We use the NASDAQ 100 stock index data downloaded from the Chicago Mercantile Exchange. Fig. 3.1 shows the cumulative distribution of daily log-returns of the stock index from January 1998 to December 2000.

The sample mean, standard deviation, skewness and kurtosis are computed as follows:

\begin{tabular}{||c|c|c|c||}
\hline Mean & Standard Deviation & Skewness & Kurtosis \\
\hline \hline 0.0007 & 0.0089 & -0.3923 & 3.8207 \\
\hline
\end{tabular}

With these statistics, we solved the mean square optimal hedging problem to compute the price of a European call option. We first note that the standard model (i.e., the Black-Scholes model) uses the information up to the second cumulant (i.e., standard deviation) only. We compare the MSOH solution with higher order cumulants with the standard Black-Scholes solution. To understand the difference between the two, we computed the implied volatility for European call options with different maturities and strike prices. Figs. $3.2-3.5$ are our numerical results, where the implied volatilities are plotted versus the strike prices (denoted by $K$ ) normalized by the initial price of the stock index, i.e., $K / S_{0}$, where the dashed is the annualized standard deviation. Each figure has a different time 


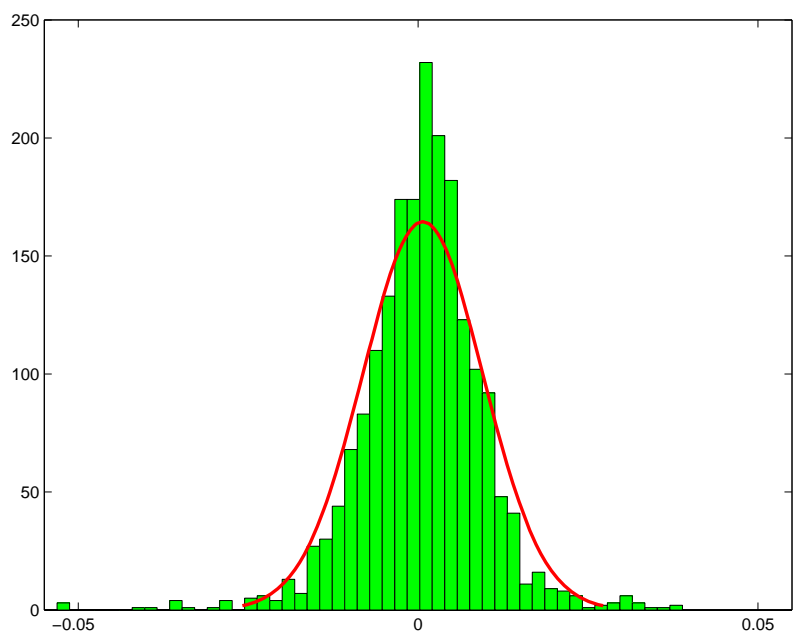

Fig. 3.1: Cumulative distribution of daily log-return

to expiration: Fig. 3.2 has an expiration of 10 days, Fig. 3.3, 20 days, Fig. 3.4, 40 days, and Fig. 3.5, 80 days. Note that the dashed line in each figure is a constant volatility corresponding to the Black-Scholes solution.

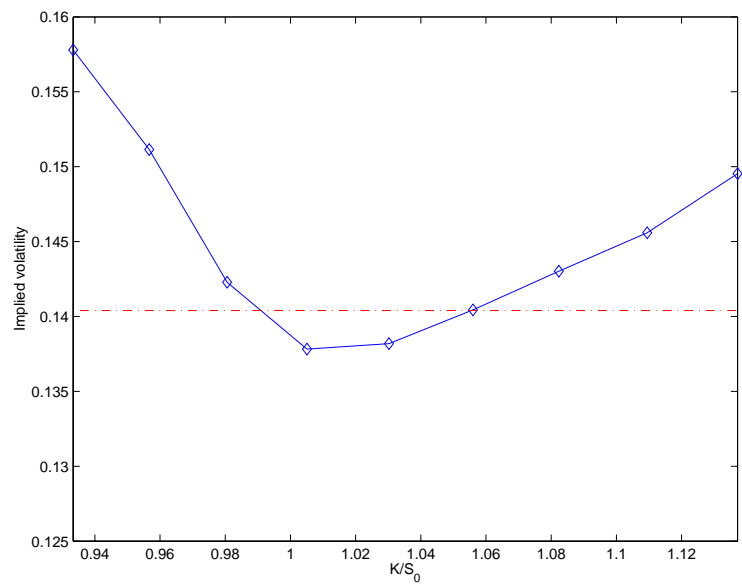

Fig. 3.2: 10 days expiration

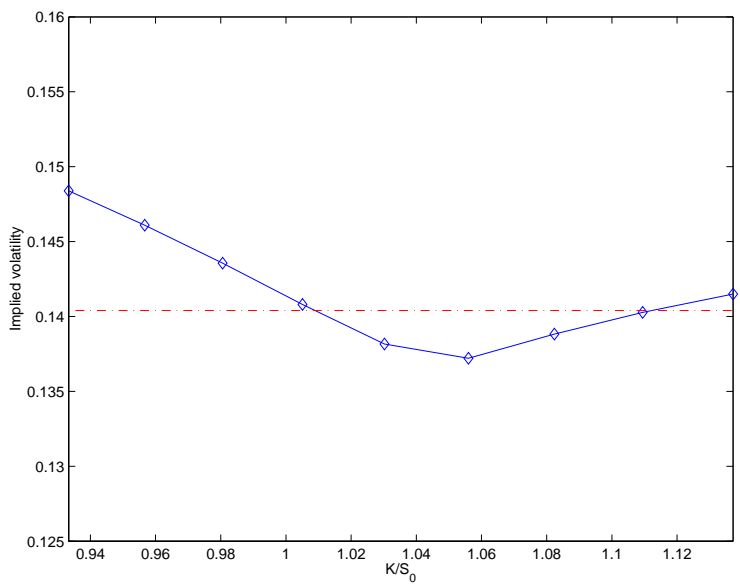

Fig. 3.3: 20 days expiration

First, we note that the smile effect is most clearly observed when the maturity is shortest. The smile effect slowly disappears as we have longer maturities, but a smirk effect remains. This can be explained using the term structure of skewness vs. smirk and the term structure of kurtosis vs. smile as follows.

Let $c_{k}$ be the $k$-th order cumulant of daily log-returns of the stock index, and let $s$ and $\kappa$ be the corresponding skewness and kurtosis, respectively. Note that the first and second order cumulants are the mean and variance, respectively. Moreover, skewness and kurtosis are functions of cumulants, which are given as follows:

$$
s=\frac{c_{3}}{c_{2}^{3 / 2}}, \quad \kappa=\frac{c_{3}}{c_{2}^{2}}
$$




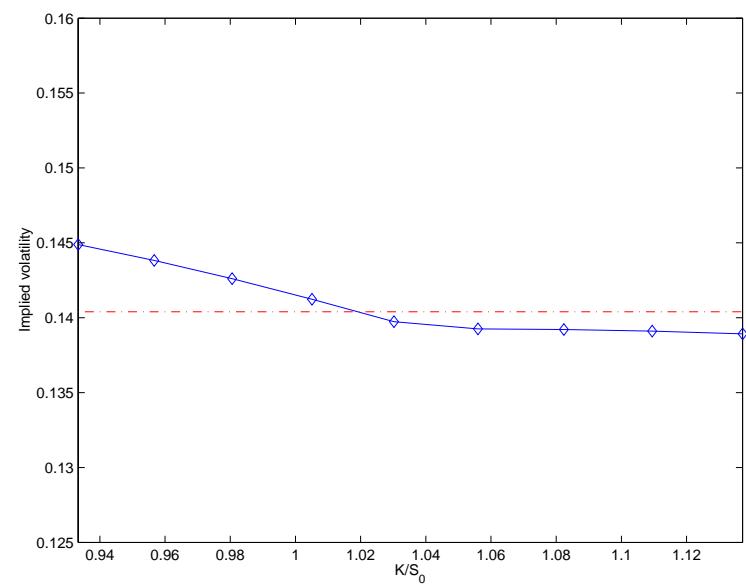

Fig. 3.4: 40 days expiration

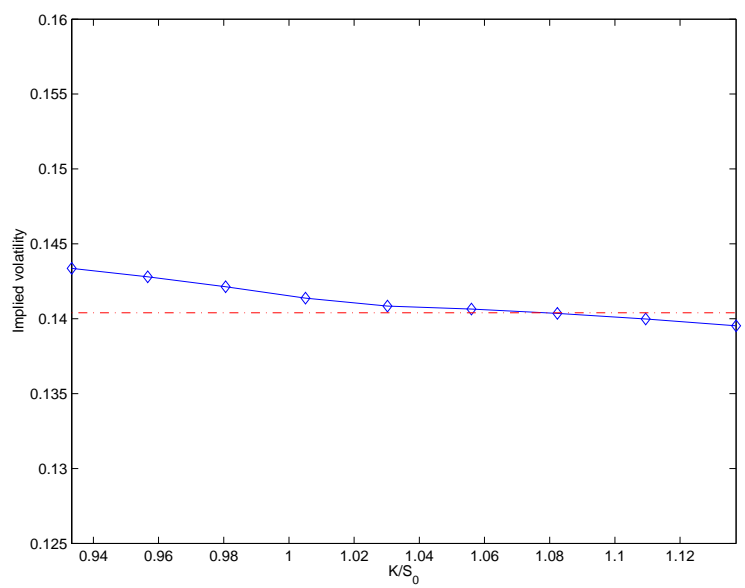

Fig. 3.5: 80 days expiration

Now, assume that the log-returns of the stock index at each day are independent. Since the cumulants have the additive property when independent random variables are summed, the $k$-th order cumulant of the $N$ day $\log$ stock return is given by

$$
N c_{k}, \quad k=1,2, \ldots
$$

If we substitute equation (3.4) to (3.3), we obtain

$$
\begin{aligned}
\text { "N day skewness" } & =\frac{N c_{3}}{\left(N c_{2}\right)^{3 / 2}}=\frac{s}{N^{1 / 2}}, \\
& N \text { day kurtosis" }=\frac{N c_{3}}{\left(N c_{2}\right)^{2}}=\frac{\kappa}{N} .
\end{aligned}
$$

Equations (3.5) and (3.6) provide the term structure of skewness and kurtosis for $N$ days with respect to daily skewness and kurtosis.

In the Black-Scholes setting where the distribution of log-stock return is given by a Gaussian distribution, skewness and kurtosis are both zero and the implied volatility is constant. On the other hand, non-zero skewness and kurtosis may increase the smirk and the smile effects as observed from our numerical experiments. Note that this result is consistent with the one described in [9] using the risk neutral probability measure, whereas our numerical experiments indicate that a similar effect is observed in the MSOH problem setting.

\section{Properties of multinomial lattices}

Here we provide further discussion about the multinomial lattice with five branches given in Section 2. Note that the relation between the multinomial lattice parameterization and the finite difference method for partial differential equations is compared in Appendix A. In this section, we explore the limiting property of the lattice model. To this end, we show the following proposition:

Proposition 2 The following two conditions hold:

1. If skewness and kurtosis at each time period $\tau$ are fixed, then the distribution of the multinomial lattice converges to a Gaussian. 
2. If annualized skewness and kurtosis are fixed, then the distribution of the multinomial lattice converges to the one given by a compound Poisson process with the same skewness and kurtosis.

Remark 2 Note that the convergence in Proposition 2 is in distribution of a random variable defined by the multinomial lattice when $\tau \rightarrow 0$, i.e., the distribution of the multinomial lattice converges to the one given by a Gaussian process or a compound Poisson process for any fixed time $t_{N}$.

Let $X_{0}=0$ without loss of generality. Similar to (3.4), the $k$-th order cumulant of

$$
X_{N}=\sum_{n=0}^{N-1} X_{n+1}-X_{n}
$$

is given by $N c_{k}$, where $c_{k}$ is the $k$-th order cumulant of $X_{n+1}-X_{n}$. Because the first and the second order cumulants are mean and variance, mean and variance of $X_{N}$ are, respectively, given as

$$
N c_{1} \text { and } N c_{2} \text {. }
$$

To make mean and variance of $X_{N}$ finite when $N \rightarrow \infty$, assume that annualized mean and variance (i.e., mean and variance of $X_{N}$ when $t_{N}=1$ ) are given as $\hat{\nu}<\infty$ and $\hat{\sigma}^{2}<\infty$. In this case, mean and variance of $X_{n+1}-X_{n}$ at a basic time period may be given as

$$
c_{1}=\hat{\nu} \tau, \quad c_{2}=\hat{\sigma}^{2} \tau
$$

respectively. We consider the following two cases:

\subsection{Case 1: Fixed skewness and kurtosis of a basic time period}

For any given $T>0$, let $t_{N}=T$. We will show that the distribution of $X_{N}$ converges to a Gaussian distribution given as

$$
\mathcal{N}\left(\hat{\nu} T, \hat{\sigma}^{2} T\right)
$$

when $N \rightarrow \infty$, where $\hat{\nu}$ and $\hat{\sigma}^{2}$ are annualized mean and variance. Note that this is a consequence of the central limit theorem for i.i.d. random variables.

Let $\alpha$ be given as in (2.16) for some $\beta$ such that $p_{i}, i=1, \ldots, 5$, are positive, and assume that skewness and kurotsis, $s$ and $\kappa$, at a basic time period are fixed. Since $\sigma=\hat{\sigma} \sqrt{\tau}, \alpha$ is written as

$$
\alpha=\hat{\alpha} \sqrt{\tau},
$$

where

$$
\hat{\alpha}=\hat{\sigma} \sqrt{\frac{3+\kappa}{\beta}}
$$

Define $Y_{n}$ by

$$
Y_{n}:=\sqrt{N}\left(X_{n}-X_{n-1}-\hat{\nu} \tau\right), \quad n=1, \ldots, N .
$$

We can readily verify that $Y_{n}$ takes one of the following states

$$
(5-2 l+1) \hat{\alpha} \sqrt{T}, \quad l=1, \ldots, 5
$$


with probabilities $p_{l}, l=1, \ldots, 5$, respectively. Noting that $p_{l}, l=1, \ldots, 5$, and $\hat{\alpha}$ are fixed if $s, \kappa$, and $\beta$ are given, $Y_{n}$ is a sequence of i.i.d. random variables. Therefore, one can apply the central limit theorem for i.i.d. random variables to show the following:

$$
\lim _{N \rightarrow \infty} \frac{1}{\sqrt{N}} \sum_{n=1}^{N} Y_{n} \sim \mathcal{N}\left(0, \hat{\sigma}^{2} T\right)
$$

Condition (4.7) is equivalent to

$$
\lim _{N \rightarrow \infty} \sum_{n=1}^{N}\left(X_{n}-X_{n-1}\right) \sim \mathcal{N}\left(\hat{\nu} T, \hat{\sigma}^{2} T\right)
$$

Hence, we see that the distribution of $X_{N}$ converges to (4.3).

\subsection{Case 2: Fixed annualized skewness and kurtosis}

Here we consider the case where $\alpha$ is chosen as in (2.23), or $\beta=12$ in (2.16). Note that a similar result may be obtained by choosing a different $\beta$ as long as the probabilities $p_{1}, \ldots, p_{5}$ are positive. We will show that the following lemma holds:

Lemma 1 The distribution of the multinomial lattice converges to the distribution of the following compound Poisson process when annualized skewness and kurtosis, $\hat{s}$ and $\hat{\kappa}$, are fixed:

$$
\mathrm{d} X(t)=\hat{\nu} \mathrm{d} t+W \mathrm{~d} Y(t)
$$

where $Y(t)$ is a Poisson process with intensity

$$
\lambda:=\frac{3}{2 \hat{\kappa}}
$$

and $W$ is a jump size which takes one of the following values

$$
(6-2 i) \hat{\sigma} \sqrt{\frac{\hat{\kappa}}{12}}, \quad i=1,2,4,5
$$

with probabilities

$$
q_{1}=\frac{1}{6}\left(1+\hat{s} \sqrt{\frac{3}{\hat{\kappa}}}\right), q_{2}=\frac{1}{3}\left(1-\hat{s} \sqrt{\frac{3}{\hat{\kappa}}}\right), q_{4}=\frac{1}{3}\left(1+\hat{s} \sqrt{\frac{3}{\hat{\kappa}}}\right), q_{5}=\frac{1}{6}\left(1-\hat{s} \sqrt{\frac{3}{\hat{\kappa}}}\right)
$$

at each time $t \in\left[0, t_{N}\right]$.

We will show that the moment generating function of $X_{N}$ converges to the one for the compound Poisson process in Lemma 1, which is given as

$$
\psi(v)=e^{\hat{\nu} T v+\lambda T \mathbb{E}\left(e^{v W}-1\right)}
$$

for any given $T=t_{N}$. Let us replace $s$ and $\kappa$ in (2.25) with the ones using the annualized skewness $\hat{s}$ and kurtosis $\hat{\kappa}$, i.e.,

$$
s=\frac{\hat{s}}{\sqrt{\tau}}, \quad \kappa=\frac{\hat{\kappa}}{\tau}
$$


Then we have

$$
\left.\begin{array}{l}
{\left[p_{1}, p_{2}, p_{3}, p_{4}, p_{5}\right]} \\
=\left[\frac{3+\frac{\hat{\kappa}}{\tau}+\frac{\hat{s}}{\sqrt{\tau}} \sqrt{9+\frac{3 \hat{\kappa}}{\tau}}}{4\left(3+\frac{\hat{\kappa}}{\tau}\right)^{2}}, \frac{3+\frac{\hat{\kappa}}{\tau}-\frac{\hat{s}}{\sqrt{\tau}} \sqrt{9+\frac{3 \hat{\kappa}}{\tau}}}{2\left(3+\frac{\hat{\kappa}}{\tau}\right)^{2}}, \frac{3+2 \frac{\hat{\kappa}}{\tau}}{2\left(3+\frac{\hat{\kappa}}{\tau}\right)},\right. \\
\left.\frac{3+\frac{\hat{\kappa}}{\tau}+\frac{\hat{s}}{\sqrt{\tau}} \sqrt{9+\frac{3 \hat{\kappa}}{\tau}}}{2\left(3+\frac{\hat{\kappa}}{\tau}\right)^{2}}, \frac{3+\frac{\hat{\kappa}}{\tau}-\frac{\hat{s}}{\sqrt{\tau}} \sqrt{9+\frac{3 \hat{\kappa}}{\tau}}}{4\left(3+\frac{\hat{\kappa}}{\tau}\right)^{2}}\right] \\
=\left[\frac{3 \tau+\hat{\kappa}+\hat{s} \sqrt{9 \tau+3 \hat{\kappa}}}{4(3 \tau+\hat{\kappa})^{2}}, \frac{3 \tau+\hat{\kappa}-\hat{s} \sqrt{9 \tau+3 \hat{\kappa}}}{2(3 \tau+\hat{\kappa})^{2}}, 1-\frac{3 \tau}{2(3 \tau+\hat{\kappa})},\right. \\
\left.\frac{3 \tau+\hat{\kappa}+\hat{s} \sqrt{9 \tau+3 \hat{\kappa}}}{2(3 \tau+\hat{\kappa})^{2}} \tau, \frac{3 \tau+\hat{\kappa}-\hat{s} \sqrt{9 \tau+3 \hat{\kappa}} \tau}{4(3 \tau+\hat{\kappa})^{2}}\right]
\end{array}\right]
$$

and

$$
\alpha=\hat{\sigma} \sqrt{\frac{3 \tau+\hat{\kappa}}{12}}
$$

Let $\lambda_{\tau}$ be defined as

$$
\lambda_{\tau}:=\frac{3}{2(3 \tau+\hat{\kappa})},
$$

and rewrite the last equation in (4.15) as

$$
\left[p_{1}, p_{2}, p_{3}, p_{4}, p_{5}\right]=\left[\lambda_{\tau} \tau q_{\tau, 1}, \lambda_{\tau} \tau q_{\tau, 2}, 1-\lambda_{\tau} \tau, \lambda_{\tau} \tau q_{\tau, 4}, \lambda_{\tau} \tau q_{\tau, 5}\right]
$$

where

$\left[q_{\tau, 1}, q_{\tau, 2}, q_{\tau, 4}, q_{\tau, 5}\right]$

$$
=\left[\frac{1}{6}\left(1+\hat{s} \sqrt{\frac{3}{3 \tau+\hat{\kappa}}}\right), \frac{1}{3}\left(1-\hat{s} \sqrt{\frac{3}{3 \tau+\hat{\kappa}}}\right), \frac{1}{3}\left(1+\hat{s} \sqrt{\frac{3}{3 \tau+\hat{\kappa}}}\right), \frac{1}{6}\left(1-\hat{s} \sqrt{\frac{3}{3 \tau+\hat{\kappa}}}\right)\right] .
$$

Note that $q_{\tau, 1}, q_{\tau, 2}, q_{\tau, 4}, q_{\tau, 5}$ in (4.19) may be considered as probabilities since

$$
q_{\tau, 1}+q_{\tau, 2}+q_{\tau, 4}+q_{\tau, 5}=1, \quad q_{\tau, 1}, q_{\tau, 2}, q_{\tau, 4}, q_{\tau, 5}>0 .
$$

Let $W_{n}(\tau)$ be a random variable which takes one of the following states

$$
(6-2 i) \alpha, \quad i=1,2,4,5 .
$$

with probabilities $q_{\tau, i}, i=1,2,4,5$, at time $t_{n}, n=1, \ldots, N$. Also let $Y_{n}(\tau)$ be a random variable which takes, at time $t_{n}, n=1, \ldots, N$, either 0 with probability $1-\lambda \tau$ or 1 with probability $\lambda \tau$, and is independent of $W_{n}(\tau)$. Then $X_{n}-X_{n-1}$ may be written using the product of two random variables as follows:

$$
X_{n}-X_{n-1}=\hat{\nu} \tau+W_{n}(\tau) Y_{n}(\tau)
$$

Consider the $\log$ of the moment generating function of $X_{N}$, i.e.,

$$
\ln \psi_{N}(v)=\ln \left[\mathbb{E}\left(e^{v X_{N}}\right)\right], \quad X_{N}=\sum_{n=1}^{N}\left(X_{n}-X_{n-1}\right)=\hat{\nu} T+\sum_{n=1}^{N} W_{n}(\tau) Y_{n}(\tau) .
$$


Since $X_{n}-X_{n-1}, n=1, \ldots, N$, are i.i.d., and $W_{n}(\tau)$ and $Y_{n}(\tau)$ are independent, we have the following:

$$
\begin{aligned}
\ln \psi_{N}(v) & =\ln \mathbb{E}\left[e^{\hat{\nu} T v+\sum_{n=1}^{N} v W_{n}(\tau) Y_{n}(\tau)}\right] \\
& =\hat{\nu} T v+\sum_{n=1}^{N} \ln \left[\mathbb{E}\left(e^{v W_{n}(\tau) Y_{n}(\tau)}\right)\right] \\
& =\hat{\nu} T v+N \ln \left[\mathbb{E}\left(e^{v W_{1}(\tau) Y_{1}(\tau)}\right)\right] \\
& =\hat{\nu} T v+N \ln \left[\lambda_{\tau} \tau \mathbb{E}\left(e^{v W_{1}(\tau)}\right)+1-\lambda_{\tau} \tau\right]
\end{aligned}
$$

Let $\xi_{\tau}$ be defined as

$$
\xi_{\tau}:=\mathbb{E}\left(e^{v W_{1}(\tau)}\right)
$$

Note that

$$
\lim _{\tau \rightarrow 0} \xi_{\tau}=\lim _{\tau \rightarrow 0} \sum_{i=1,2,4,5} q_{\tau, i} e^{v(6-2 i) \alpha}=\mathbb{E}\left(e^{v W}\right) .
$$

The last condition in (4.23) may be rewritten as

$$
\hat{\nu} T v+N \ln \left[\lambda_{\tau} \tau \mathbb{E}\left(e^{v W_{1}(\tau)}\right)+1-\lambda_{\tau} \tau\right]=\hat{\nu} T v+\frac{1}{\tau} T \ln \left[\lambda_{\tau} \tau \xi_{\tau}+1-\lambda_{\tau} \tau\right]
$$

Now we take the limit as $N \rightarrow \infty$, or $\tau \rightarrow 0$, as

$$
\begin{aligned}
\lim _{N \rightarrow \infty} \ln \psi_{N}(v) & =\hat{\nu} T v+\lim _{\tau \rightarrow 0} \frac{\ln \left[\lambda_{\tau} \tau \xi_{\tau}+1-\lambda_{\tau} \tau\right]}{\tau} \cdot T \\
& =\hat{\nu} T v+\lim _{\tau \rightarrow 0} \frac{\left(\ln \left[\lambda_{\tau} \tau \xi_{\tau}+1-\lambda_{\tau} \tau\right]\right)^{\prime}}{\tau^{\prime}} \cdot T \\
& =\hat{\nu} T v+\lim _{\tau \rightarrow 0} \frac{\left(\lambda_{\tau} \tau \xi_{\tau}+1-\lambda_{\tau} \tau\right)^{\prime}}{\lambda_{\tau} \tau \xi_{\tau}+1-\lambda_{\tau} \tau} \cdot T \\
& =\hat{\nu} T v+\lim _{\tau \rightarrow 0}\left(\lambda_{\tau} \xi_{\tau}+\lambda_{\tau}^{\prime} \tau \xi_{\tau}+\lambda_{\tau} \tau \xi_{\tau}^{\prime}-\lambda_{\tau}-\lambda_{\tau}^{\prime} \tau\right) \cdot T \\
& =\hat{\nu} T v+\lambda T \mathbb{E}\left(e^{v W}-1\right) \\
& =\ln \psi(v) .
\end{aligned}
$$

Consequently, we conclude that

$$
\lim _{N \rightarrow \infty} \psi_{N}(v)=\psi(v)
$$

\section{Comparison with jump-diffusion model}

We have shown in the previous section that the distribution of the multinomial lattice converges to either a Gaussian or a compound Poisson when the basic time period approaches zero. On the other hand, the so-called jump-diffusion process consists of a Gaussian part and a compound Poisson part, and one may be interested in the difference and/or similarity between the multinomial lattice and jump-diffusion models. For this reason, we are going to compare with a jump-diffusion model, although there are a variety of Levy processes that possess non-zero skewness and excess kurtosis. For simplicity, we consider the case where the stock process is already risk neutralized for both the jump-diffusion and multinomial lattice model, and assume that they have the same mean, variance, skewness, and kurtosis under the risk neutral probability measure. 
Here we apply the risk neutral pricing formula for jump-diffusion models in [24], where the log of the underlying asset price, $X(t)=\ln S(t), t \in\left[0, t_{N}\right]$, is given by the following stochastic differential equation under the risk neutral measure:

$$
\mathrm{d} X(t)=\left(r-\frac{\sigma^{2}}{2}-\lambda \nu_{J}\right) \mathrm{d} t+\sigma_{B} \mathrm{~d} B(t)+J \mathrm{~d} Y(t)
$$

where $Y(t)$ is a Poisson process with intensity $\lambda$ and $B(t)$ is the standard Brownian motion. $J$ is the jump size which we assume follows another Gaussian distribution

$$
J \sim \mathcal{N}\left(\nu_{J}, \sigma_{J}^{2}\right)
$$

at each time $t \in\left[0, t_{N}\right]$.

We use the following parameter set:

$$
S_{0}=100, \quad r=0.06, \quad \sigma_{B}=0.2, \quad \nu_{J}=-0.05, \quad \sigma_{J}=.1, \quad \lambda=0.05
$$

We compute the implied volatility for European call options with different maturities (10 days, 20 days and 40 days) and strike prices. Fig. 5.1-5.3 show our numerical results, where the implied volatilities are plotted versus the strike prices normalized by the initial price of the stock (i.e., $\left.K / S_{0}\right)$. The dashed line in each figure is a constant volatility corresponding to the annualized standard deviation. From this numerical experiment, one can observe the term structure of implied volatility and note that the shorter maturity provides the clearer smile effect.

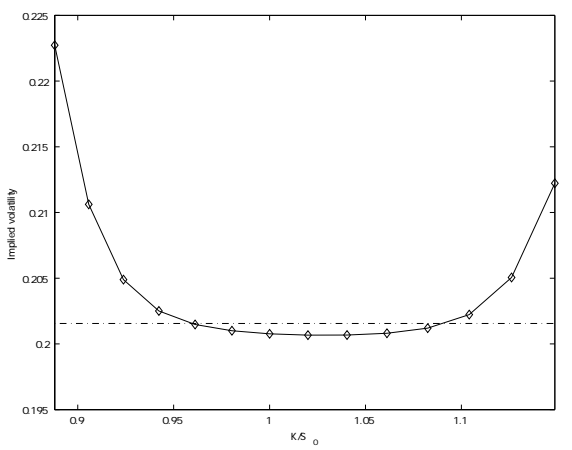

Fig. 5.1: 10 days expiration

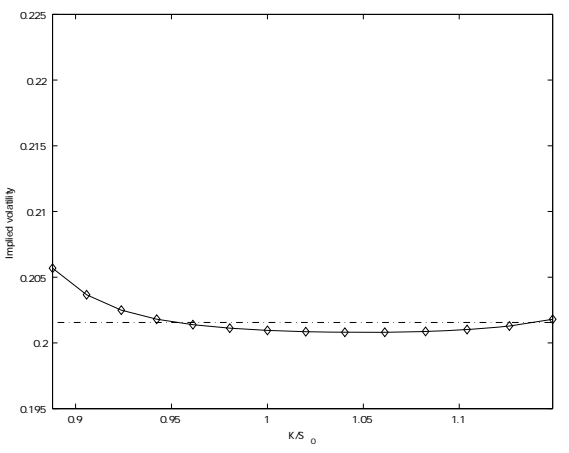

Fig. 5.2: 20 days expiration

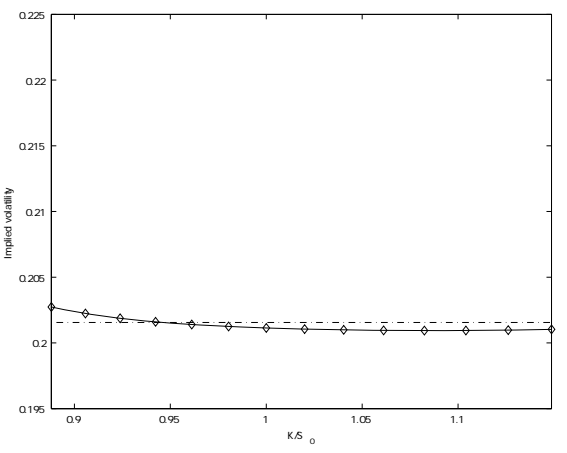

Fig. 5.3: 40 days expiration

Next, we compute the risk neutral prices, where the following standard risk neutral pricing formula can be applied if the underlying stock process is modeled on a multinomial lattice under the risk neutral probability measure. By using an appropriate discount factor, the initial price of a European derivative security is obtained as

$$
V_{0}=(1+r)^{-N} \tilde{\mathbb{E}}\left(V_{N}\right)
$$

where $\tilde{\mathbb{E}}$ is the expectation under the risk neutral probability measure and $r$ is a fixed risk free rate. Equation (5.3) can be solved using the standard backward calculation to find $V_{0}$.

We constructed a multinomial lattice using the same skewness and kurtosis as the above jumpdiffusion example, where four cumulants were matched using five branches. The skewness and kurtosis were computed as

$$
s=-0.16, \quad \kappa=3.5
$$




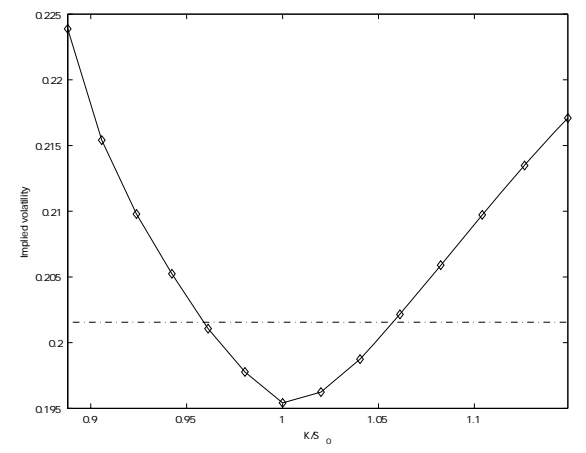

Fig. 5.4: 10 days expiration

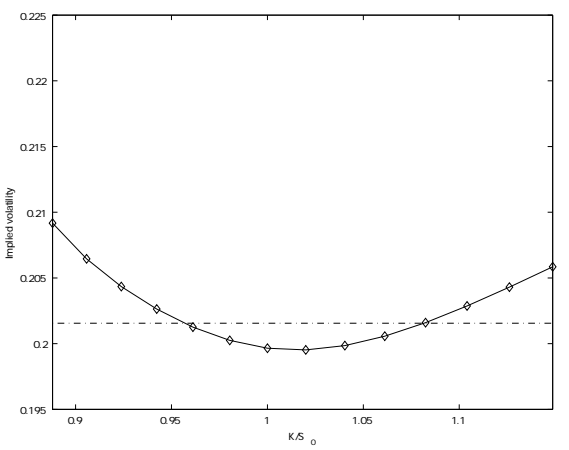

Fig. 5.5: 20 days expiration

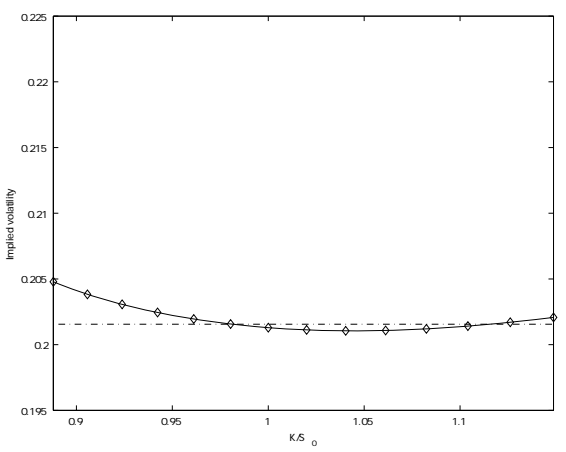

Fig. 5.6: 40 days expiration

to match the ones given by the jump-diffusion model. Similar to the previous example, we computed implied volatility surfaces, and obtained Fig. 5.4-5.6. Note that even with the same skewness and kurtosis under the risk neutral probability measure, the multinomial lattice model exhibits clearer implied volatility surfaces. It might be fair to say that this result is more consistent with real market behavior since the volatility smile effect in the real market tends to be more pronounced than the one obtained from the jump-diffusion model, and additionally the multinomial lattice model addresses the specific influence of skewness and kurtosis more directly.

Finally, we computed implied volatility surfaces when we match up to the 5th and 6 th cumulants using a multinomial lattice, in addition to mean, variance, skewness, and kurtosis in the jump-diffusion model, and obtained Fig. 5.7. In this case, we see that the implied volatility surfaces are closer to the ones obtained from the jump-diffusion model.

Fig. 5.7: Multinomial lattice with the first 6 cumulants

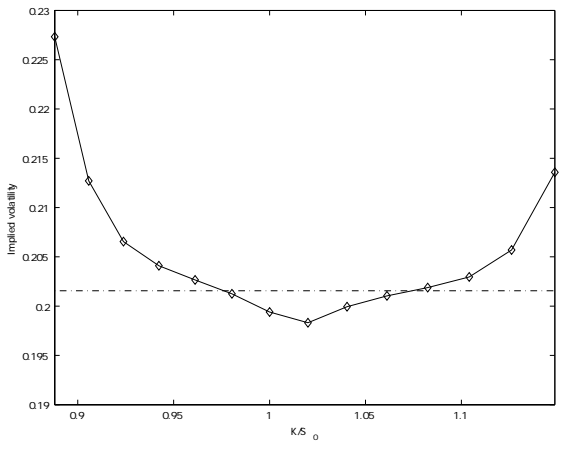

10 days expiration

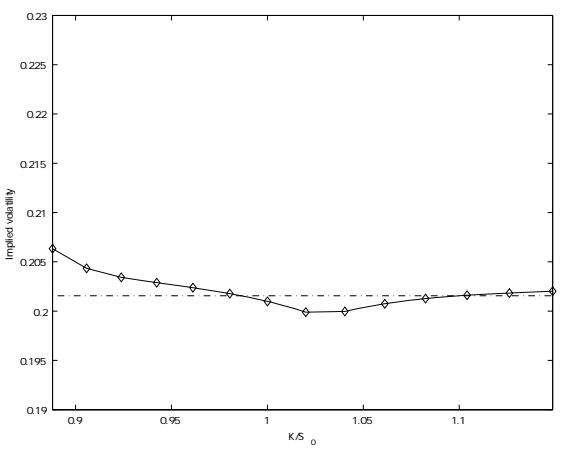

20 days expiration

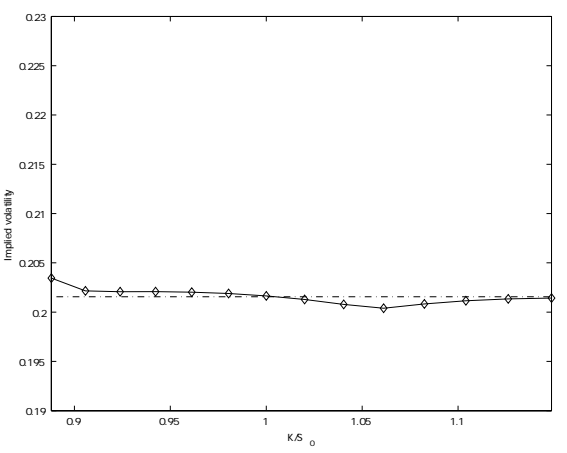

40 days expiration

\section{Conclusion}

In this paper, we showed some properties of multinomial lattices that model the underlying stock dynamics by taking into account any given cumulants. First, we provided a parameterization of multinomial lattices, and demonstrated that mean, variance, skewness, and kurtosis of the underlying may be matched using five branches. Then, we investigated the convergence of the multinomial 
lattice when the basic time period approaches zero, and proved that the limiting distribution of the multinomial lattice that matches annualized mean, variance, skewness and kurtosis is given by that of a compound Poisson process. Finally, we illustrated the effect of higher order cumulants in the underlying asset process on the price of derivative securities through numerical experiments using the multinomial lattice, and provided a comparison with jump-diffusion models.

\section{Acknowledgment}

We would like to acknowledge the anonymous reviewers for making valuable comments about the paper.

\section{References}

[1] K.I. Amin, "Jump Diffusion Option Valuation in Discrete Time," Journal of Finance, 48(5):1833$1863,1993$.

[2] M. Avellaneda, A. Levy, and Antonio Paras, "Pricing and hedging derivatives in markets with uncertain volatilities," Applied Mathematical Finance, 2:73-88, 1995.

[3] F. Black and M. Scholes, "The Pricing of Options and Corporate Liabilities," Journal of Political Economy, 81:637-654, 1973.

[4] P. Carr and L. Wu, "The finite moment logstable process and option pricing," Journal of Finance, 58:753-778, 1993.

[5] R. Cont and E. Voltchkova, "A Finite Difference Scheme for Option Pricing in Jump Diffusion and Exponential Levy Models," to appear in SIAM Journal of Numerical Analysis.

[6] C.J. Corrado and T. Su, "Skewness and Kurtosis in S\&P 500 Index Returns Implied by Option Prices," Journal of Financial Research, 19:175-192, 1996.

[7] J.C. Cox and S.A. Ross, "The valuation of options for alternative stochastic processes," Journal of Financial Economics, 3:145-166, 1976.

[8] J.C. Cox, S.A. Ross, and M. Rubinstein, "Option pricing: A simplified approach," Journal of Financial Economics, 7:229-263, 1979.

[9] S.R. Das and R.K. Sundaram, "Of Smiles and Smirks: A Term-Structure Perspective," Research report, 1998.

[10] E. Derman and I. Kani, "Implied Trinomial Trees of the Volatility Smile," Goldman Sachs Quantitative Strategies Research Notes, February, 1994.

[11] D. Duffie and H.R. Richardson, "Mean-variance hedging in continuous time," Annals Appl. Probability, 1, 1-15, 1991.

[12] B. Dupire, "Pricing with a Smile," Risk, 7(1):18-20, 1994. 
[13] S. Fedotov and S. Mikhailov, "Option Pricing for Incomplete Markets via Stochastic Optimization: Transaction Costs, Adaptive Control, and Forecast," Int. J. of Theoretical and Applied Finance, 4(1):179-195, 2001.

[14] H. Follmer and M. Schweizer, "Hedging of contingent claims under incomplete information", In Applied Stochastic Analysis (M.H.A. Davis and R.J. Elliott, eds.), Stochastics Monographs 5, 389-414. Gordon and Breach, New York, 1991.

[15] O. Hammarlid, "On minimizing risk in incomplete markets option pricing models," Int. J. Theoretical and Applied Finance, 1(2):227-233, 1998.

[16] H. Geman, D.B. Madan, and M. Yor, "Time changes for Levy processes," Mathematical Finance, 11:79-96, 2001.

[17] J. Hull and A. White, "The Pricing of Options on Assets with Stochastic Volatilities," Journal of Finance, 42:281-300, 1987.

[18] J. Hull and A. White, "Valuing Derivative Securities Using the Explicit Finite Difference Method," Journal of Financial and Quantitative Analysis, 25:87-100, 1990.

[19] J. Hull and A. White, "Numerical procedures for implementing term structure models I," Journal of Derivatives, fall:7-16, 1994.

[20] R. Jarrow and A. Rudd, "Approximate option valuation for arbitrary stochastic processes," Journal of Financial Economics, 10:347-369, 1982.

[21] R.L. Karandikar and S.T. Rachev, "A generalized binomial model and option pricing formulae for subordinated stock-price processes," Probability and Mathematical Statics, 15:427-447, 1995.

[22] S.G. Kou, "A Jump-Diffusion Model for Option Pricing," Management Science, 48(8):1086-1101, 2002.

[23] A. Li, P. Ritchken, and L. Sankarasubramanian, "Lattice Methods for Pricing American Interest Rate Claims," Journal of Finance, 50:719-737, 1995.

[24] R.C. Merton, "Option pricing when underlying stock returns are discontinuous," Journal of Financial Economics, 3:125-144, 1976.

[25] Y. Miyahara (2001), "[Geometric Levy Process \& MEMM] Pricing Model and Related Estimation Problems," Asia-Pacific Financial Markets, 8(1):45-60.

[26] C.D. Pirkner, A.S. Weigend and H. Zimmermann, "Extracting Risk-Neutral Densities from Option Prices Using Mixture Binomial Trees," Proc. of the 1999 IEEE/IAFE/INFORMS Conf. on Computational Intelligence for Financial Engineering, 135-158, 1999.

[27] M. Potters, R. Cont and J-P Bouchaud, "Financial markets as adaptive systems," Europhys. Lett., 41(3):239-244, 1998.

[28] J.A. Primbs and Y. Yamada, "A Moment based Analysis of Hedging under Discrete Trading," Proc. of the 2003 International Conference on Computational Intelligence for Financial Engineering (CIFEr), 2003. 
[29] M. Rubinstein, "Implied Binomial Trees," Journal of Finance, 3:771-818, 1994.

[30] M. Rubinstein, "Edgeworth Binomial Trees," Journal of Derivatives, 1998.

[31] R.J. Ritchey, "Call Option Valuation for Discrete Normal Mixtures," Journal of Financial Research, 4:285-296, 1990.

[32] M. Schäl, "On quadratic cost criteria for option hedging," Math. Oper. Res., 19:121-131, 1994.

[33] M. Schweizer, "Variance-optimal hedging in discrete time," Math. Oper. Res., 20:1-32, 1995.

[34] Y. Yamada and J.A. Primbs, "Construction of Multinomial Lattice Random Walks for Optimal Hedges," Proc. of the 2001 International Conference of Computational Science, 579-588, 2001.

[35] Y. Yamada and J.A. Primbs, "Mean Square Optimal Hedges Using Higher Order Moments," Proc. of the 2003 International Conference on Computational Intelligence for Financial Engineering (CIFEr), 2003.

\section{Appendix}

\section{A Cumulant based finite difference approximations}

The objective of this subsection is to compare the multinomial lattice parameterization with the finite difference method for a certain partial differential equation (PDE). We first note that the explicit finite difference method of the standard Black-Scholes PDE is known to be equivalent to the trinomial lattice [18]. Therefore, one may wonder what is equivalent to the multinomial lattice provided in Section 2. In order to consider this question, we will introduce a general Black-Scholes type PDE with cumulants as follows.

\section{A.1 Black-Scholes type PDE with cumulants}

Let $X(t), t \in[0, T]$, be a (continuous time) Levy process on a probability space $(\Omega, \mathcal{F}, \mathbb{P})$. For simplicity, we assume that $X(t)$ is a stationary process with finite moments, and also let $c_{k}(k=$ $1,2, \ldots)$ be the $k$-th order annualized cumulant of $X(t)$, i.e., the cumulant of $X(1)$. From the additive property of cumulants, the $k$-th order cumulant of $X(t)$ is given by $c_{k} t$ and the characteristic function of $X(t)$ by

$$
\mathbb{E}\left[e^{s X(t)}\right]=\exp \left(\sum_{k=1}^{\infty} \frac{c_{k} t}{k !} s^{k}\right) .
$$

Moreover, the conditional characteristic function of $X(T), T>t$ given $x(t)=x$, denoted by $\phi(x, t)$, is written as

$$
\begin{aligned}
\phi(x, t) & :=\mathbb{E}\left[e^{s(X(T)-X(t))} \mid X(t)=x\right] \\
& =e^{s x} \exp \left(\sum_{k=1}^{\infty} \frac{c_{k}(T-t)}{k !} s^{k}\right) .
\end{aligned}
$$

It is readily confirmed that $\phi(x, t)$ is the solution to the following Kolmogorov Backward equation:

$$
-\frac{\partial}{\partial t} \phi(x, t)=\sum_{k=1}^{\infty} \frac{c_{k}}{k !} \frac{\partial^{k}}{\partial x^{k}} \phi(x, t), \quad \phi(x, T)=e^{s x} .
$$


Let $g(\cdot)$ be a payoff function which is Borel measurable on $(\Omega, \mathcal{F}, \mathbb{P})$, and define

$$
u(x, t):=\mathbb{E}(g(X(T)) \mid X(t)=x) .
$$

Then a standard argument shows that $u(x, t)$ satisfies the following:

$$
-\frac{\partial}{\partial t} u(x, t)=\sum_{k=1}^{\infty} \frac{c_{k}}{k !} \frac{\partial^{k}}{\partial x^{k}} u(x, t), u(x, T)=g(x) .
$$

Denote the discounted process of $u(x, t)$ by $v(x, t)$, where

$$
v(x, t):=e^{r(T-t)} u(x, t) .
$$

By applying the Feynman-Kac theorem to the discounted process $v(x, t)$, the PDE in (A.5) is modified as follows:

$$
r v(x, t)=\frac{\partial}{\partial t} v(x, t)+\sum_{k=1}^{\infty} \frac{c_{k}}{k !} \frac{\partial^{k}}{\partial x^{k}} v(x, t), \quad v(x, T)=g(x) .
$$

The above equation addresses the standard Black-Scholes equation in log-coordinate as a special case. To see this, assume that $X(t)$ is the log return of the underlying stock price $S(t)$, i.e., $X(t)=$ $\ln \frac{S(t)}{S(0)}$, which satisfies

$$
\mathrm{d} X(t)=\left(r-\frac{\sigma^{2}}{2}\right) \mathrm{d} t+\sigma \mathrm{d} B(t)
$$

where $B(t)$ is a Brownian motion under the risk neutral probability measure. Then, we have the Black-Scholes equation for a European contingent claim in log-coordinate as follows:

$$
r v(x, t)=\frac{\partial}{\partial t} v(x, t)+\left(r-\frac{\sigma^{2}}{2}\right) \frac{\partial}{\partial x} v(x, t)+\frac{\sigma^{2}}{2} \frac{\partial^{2}}{\partial x^{2}} v(x, t), \quad v(x, T)=g(x) .
$$

Note that condition (A.8) implies that $X(T)$ is normal with mean $\left(r-\frac{\sigma^{2}}{2}\right) T$ and variance $\sigma^{2} T$, and thus, the annualized cumulants are given by

$$
c_{1}=r-\frac{\sigma^{2}}{2}, \quad c_{2}=\sigma, \quad c_{k}=0, \quad k=3,4, \ldots
$$

Therefore, we see that the Black-Scholes equation can be written as (A.7) with the cumulants in (A.10).

In Merton's jump-diffusion case under the risk neutral probability measure, the return process may be represented as

$$
\mathrm{d} X(t)=\nu \mathrm{d} t+\sigma \mathrm{d} B(t)+J \mathrm{~d} N(t)
$$

where $N(t)$ is a Poisson process with intensity parameter $\lambda$ and

$$
\nu:=r-\frac{\sigma^{2}}{2}-\lambda \mathbb{E}(J)
$$

In this case, the value process of a European contingent claim, $v(x, t)$, satisfies the following PDE:

$$
\begin{aligned}
r v(x, t) & =\frac{\partial}{\partial t} v(x, t)+\nu \frac{\partial}{\partial x} v(x, t)+\frac{\sigma^{2}}{2} \frac{\partial^{2}}{\partial x^{2}} v(x, t)+\lambda \mathbb{E}(v(x+J, t)-v(x, t)), \\
v(x, T) & =g(x) .
\end{aligned}
$$


Since

$$
v(x+J, t)-v(x, t)=\sum_{k=1}^{\infty} J^{k} \frac{\partial^{k}}{\partial x^{k}} v(x, t)
$$

holds, the PDE in (A.13) can be rewritten as

$$
r v(x, t)=\frac{\partial}{\partial t} v(x, t)+\nu \frac{\partial}{\partial x} v(x, t)+\frac{\sigma^{2}}{2} \frac{\partial^{2}}{\partial x^{2}} v(x, t)+\lambda \sum_{k=1}^{\infty} \frac{\mathbb{E}\left(J^{k}\right)}{k !} \frac{\partial^{k}}{\partial x^{k}} v(x, t), \quad v(x, T)=g(x) .
$$

Noting that the $k$-th order cumulant of $X(1)$ is given as

$$
c_{1}=r-\frac{\sigma^{2}}{2}, \quad c_{2}=\sigma^{2}+\lambda \mathbb{E}\left(J^{2}\right), \quad c_{k}=\lambda \mathbb{E}\left(J^{k}\right), \quad k=3,4, \ldots
$$

we see that condition (A.15) is consistent with (A.7) for the jump-diffusion case.

\section{A.2 Finite difference approximations}

We apply a finite difference method to discretize the PDE in (A.7), allowing us to discuss the relation between the finite difference method and multinomial lattices. We show that the multinomial lattice that matches skewness and kurtosis corresponds to the finite difference approximation with the first four cumulants.

To derive the formula, we need one more step. Let $Y(t)$ be given by

$$
Y(t):=X(t)-c_{1} t
$$

using the first order cumulant (mean) of $X(1)$. Then we have the following PDE instead of (A.7) with the state variable $y$ :

$$
r f(y, t)=\frac{\partial}{\partial t} f(y, t)+\sum_{k=2}^{\infty} \frac{c_{k}}{k !} \frac{\partial^{k}}{\partial y^{k}} f(y, t), \quad f(y, T)=g\left(y+c_{1} T\right) .
$$

Noting that

$$
v(x, t)=f\left(x-c_{1} t, t\right)
$$

we see that solving (A.18) for $f$ provides the solution to (A.7) as well.

Now, we are going to discreterize the PDE in (A.18) using the explicit finite difference method. At first, consider the case where $X(t)$ satisfies (A.8), i.e., the standard Black-Scholes case in logcoordinate. In this case, the cumulants are given as in (A.10), and condition (A.18) reduces to

$$
r f(y, t)=\frac{\partial}{\partial t} f(y, t)+\frac{c_{2}}{2} \frac{\partial^{2}}{\partial y^{2}} f(y, t), \quad f(y, T)=g\left(y+c_{1} T\right) .
$$

The difference equation derived from (A.20) for the explicit method becomes

$$
r f_{i, j}=\frac{f_{i, j+1}-f_{i, j}}{\delta_{t}}+\frac{\sigma^{2}}{2} \frac{f_{i+1, j+1}+f_{i-1, j+1}-2 f_{i, j+1}}{\delta_{y}^{2}}
$$

where $\delta_{t}>0$ and $\delta_{y}>0$, and

$f_{i, j}:=f\left(y_{i}, t_{j}\right), f_{i, j+1}:=f\left(y_{i}, t_{j}+\delta_{t}\right), f_{i+1, j+1}:=f\left(y_{i}+\delta_{y}, t_{j}+\delta_{t}\right), f_{i-1, j+1}:=f\left(y_{i}-\delta_{y}, t_{j}+\delta_{t}\right)$ 
Solving equation (A.21) with respect to $f_{i, j}$ yields

$$
f_{i, j}=\frac{1}{1+r \delta_{t}}\left(\pi_{1} f_{i-1, j+1}+\pi_{2} f_{i, j+1}+\pi_{3} f_{i+1, j+1}\right)
$$

where

$$
\pi_{1}=\pi_{3}=\frac{\sigma^{2} \delta_{t}}{2 \delta_{y}^{2}}, \quad \pi_{2}=\left(1-\frac{\sigma^{2} \delta_{t}}{\delta_{y}^{2}}\right) .
$$

The coefficients $\pi_{1}, \pi_{2}$ and $\pi_{3}$ may be thought of probabilities, and are exactly the same as $p_{1}, p_{2}$ and $p_{3}$ in the multinomial lattice with three branches (i.e., trinomial lattice), respectively, if we set $\delta_{y}=2 \alpha \sqrt{\delta_{t}}$ and $\delta_{t}=\tau$.

Next, we consider the case where we have up to the 4-th cumulant. In this case, the PDE in (A.7) becomes

$$
r v(x, t)=\frac{\partial}{\partial t} v(x, t)+c_{1} \frac{\partial^{k}}{\partial x^{k}} v(x, t)+\frac{c_{2}}{2 !} \frac{\partial^{2}}{\partial x^{2}} v(x, t)+\frac{c_{3}}{3 !} \frac{\partial^{3}}{\partial x^{3}} v(x, t)+\frac{c_{4}}{4 !} \frac{\partial^{4}}{\partial x^{4}} v(x, t), v(x, T)=g(x) .
$$

Using the finite difference method for the standard Black-Scholes case in log-coordinate as a guide, we are going to discretize the above PDE. The difference equation derived from (A.20) for the standard explicit method is given as

$$
r f_{i, j}=\frac{f_{i, j+1}-f_{i, j}}{\delta_{t}}+\frac{c_{2}}{2 !} \frac{f_{i+1, j+1}+f_{i-1, j+1}-2 f_{i, j+1}}{\delta_{y}^{2}} \frac{f_{i+1, j+1}+f_{i-1, j+1}-f_{i, j+1}}{\delta_{y}^{2}}+\frac{c_{3}}{3 !} \frac{D_{3}}{\delta_{y}^{3}}+\frac{c_{4}}{4 !} \frac{D_{4}}{\delta_{y}^{4}}
$$

Here $D_{3}$ and $D_{4}$ are difference terms involving $f_{i+2}$ and $f_{i-2}$, and

$$
\begin{gathered}
f_{i, j}:=f\left(y_{i}, t_{j}\right), f_{i+2, j+1}:=f\left(y_{i}, t_{j}+2 \delta_{t}\right), f_{i+1, j+1}:=f\left(y_{i}+\delta_{y}, t_{j}+\delta_{t}\right), \\
f_{i, j+1}:=f\left(y_{i}, t_{j}+\delta_{t}\right), f_{i-1, j+1}:=f\left(y_{i}-\delta_{y}, t_{j}+\delta_{t}\right), f_{i-2, j+1}:=f\left(y_{i}-2 \delta_{y}, t_{j}+\delta_{t}\right) .
\end{gathered}
$$

Rearrangement of (A.25) with respect to $f_{i, j}$ yields

$$
f_{i, j}=\frac{1}{1+r \delta_{t}}\left(\pi_{1} f_{i-2, j+1}+\pi_{2} f_{i-1, j+1}+\pi_{3} f_{i, j+1}+\pi_{4} f_{i+1, j+1}+\pi_{5} f_{i+2, j+1}\right)
$$

where

$$
\begin{aligned}
& \pi_{1}:=\frac{1}{96}\left(\frac{s \sigma^{3}}{\alpha^{3}}+\frac{\kappa \sigma^{4}}{4 \alpha^{4}}\right), \\
& \pi_{2}:=\frac{\sigma^{2}}{4 \alpha^{2}}+\frac{1}{96}\left(-\frac{2 s \sigma^{3}}{\alpha^{3}}-\frac{\kappa \sigma^{4}}{\alpha^{4}}\right), \\
& \pi_{3}:=1-\left(\frac{\sigma^{2}}{4 \alpha^{2}}-\frac{\kappa \sigma^{4}}{64 \alpha^{4}}\right), \\
& \pi_{4}:=\frac{\sigma^{2}}{4 \alpha^{2}}+\frac{1}{96}\left(\frac{2 s \sigma^{3}}{\alpha^{3}}-\frac{\kappa \sigma^{4}}{\alpha^{4}}\right), \\
& \pi_{5}:=\frac{1}{96}\left(-\frac{s \sigma^{3}}{\alpha^{3}}+\frac{\kappa \sigma^{4}}{4 \alpha^{4}}\right) \\
& s=\frac{c_{3}}{c_{2}^{3 / 2} \sqrt{\delta_{t}}}, \kappa=\frac{c_{4}}{c_{2}^{2} \delta_{t}}
\end{aligned}
$$

and $\delta_{y}$ is chosen to satisfy

$$
\delta_{y}=2 \alpha \sqrt{\delta_{t}}
$$


Similar to the trinomial lattice case, $\pi_{1}, \ldots, \pi_{5}$ may be thought of probabilities for a multinomial lattice with five branches. If we compare these probabilities with the ones given in (2.11), we see that the terms related to skewness $s$ and kurtosis $\kappa$ are exactly the same as the ones given by $p_{1}, \ldots, p_{5}$, respectively. Therefore, the difference comes from the terms excluding the ones related to skewness and kurtosis. To see the difference, let $s=0$ and $\kappa=0$ in (A.27). Then, $\pi_{1}, \ldots, \pi_{5}$ are given as

$$
\pi_{1}=\pi_{5}=0, \quad \pi_{2}=\pi_{4}=\frac{\sigma^{2}}{4 \alpha^{2}}, \quad \pi_{3}=\left(1-\frac{\sigma^{2} \delta_{t}}{\delta_{y}^{2}}\right) .
$$

This implies that the finite difference method with 3rd and 4th cumulants reduces the trinomial lattice if we set $s=0$ and $\kappa=0$. On the other hand, the probabilities $p_{1}, \ldots, p_{5}$ in $(2.11)$ are not zero even if $s=0$ and $\kappa=0$, and they are positive if (2.12) is satisfied. 